\title{
Implantable Polyacrylonitrile-Spermidine Electrospun Nanofibers with Sustainable ROS Scavening Capacity for Osteoarthritis (OA) Therapy
}

\section{Shangzhi Gao}

Guangxi Medical University

\section{Bingcai Long}

GuangXi University of Chinese Medicine

\section{Ying Lan}

Guangxi Medical University

\section{Yanling Liang}

Guangxi Medical University

\section{Guanhua Li}

Guangxi Medical University

\section{Xin Yang}

Guangxi Medical University

\section{Maolin He}

Guangxi Medical University

\section{Li Zheng}

Guangxi Medical University https://orcid.org/0000-0002-9666-0024

Jingping Zhong ( $\sim$ jingpingzhong@163.com )

Guangxi Medical University https://orcid.org/0000-0002-3772-9896

\section{Research Article}

Keywords: Electrospun nanofibers, Reactive oxygen species, Antioxidant, Osteoarthritis

Posted Date: February 10th, 2022

DOI: https://doi.org/10.21203/rs.3.rs-1285382/v1

License: (c) (1) This work is licensed under a Creative Commons Attribution 4.0 International License. Read Full License 


\section{Abstract}

Osteoarthritis (OA) is a major cause of mobility impairment in the elderly. Scavenging excessive reactive oxygen species (ROS) to inhibit oxidative stress damage is a theraupetic strategy for OA. However, low clearance efficiency, rapid clearance by the joint fliud, poor stability and lack of sustainability challenged most ROS scavengers. Herein, we innovatively synthesized a spermidine (SPD)-acrylonitrile (PAN) copolymer nanofiber membrane using electrostatic spinning technology for effective treatment of $O A$, wherin SPD that has potent antioxidant ability served as ROS scavenger and PAN as backbone for sustainable release of SPD. Compared with the PAN, the PAN-SPD nanofibers not only greatly improved the mechanical properties, but also exhibited the sustainability and high efficiency to scavenge ROS. Moreover, PAN-SPD nanofibers have good biocompatibility, and can effectively reduce oxidative stress damage in $\mathrm{H}_{2} \mathrm{O}_{2}$ induced chondrocytes by down-regulating the expression of pro-inflammatory factors like MMP-13, IL-6 and TNF-a while up-regulating the antioxidant factors such as (SOD, CAT, GSH). There was a dramatic reduction in OA severity after PAN-SPD nanofibrous membrane treatment in OA joints compared to the untreated group after a 8-week therapy. Overall, this study provides effective strategies and new ideas for the future clinical treatment of OA or oxidative stress-related diseases.

\section{Introduction}

Osteoarthritis $(\mathrm{OA})$ is a chronic degenerative disease common in middle age and older adults that causes joint pain and movement restrictions, severely reducing the quality of life for patients [1-3]. The pathogenesis of OA is marked by articular cartilage degeneration, formation of osteophytes, and inflammation of the synovium [4]. Notably, the inflammatory response is significantly associated with excessive ROS such as hydroxyl radicals, superoxide anions and $\mathrm{H}_{2} \mathrm{O}_{2}$ and etc., in chondrocytes, $[5,6]$, often accompanied by down-regulation of antioxidant enzymes like superoxide dismutase (SOD), catalase (CAT) and etc. [6]. The resulting oxidative stress damage to chondrocytes, which in turn causes up-regulation of matrix metalloproteinase (MMPs) secretion, up-regulation of inflammatory factors, and chondrocyte apoptosis, thereby accelerating the progression of OA [6, 7]. Therefore, to scavenge excessive ROS by using promising agents may prevent the deterioration of OA.

Spermidine (SPD) $\left[\mathrm{NH}_{2}\left(\mathrm{CH}_{2}\right)_{3} \mathrm{NH}\left(\mathrm{CH}_{2}\right)_{4} \mathrm{NH}_{2}\right]$ is a natural polyamines (PAs) found in living organisms and is a crucial factor to maintain the homeostasis of the internal and external cellular environment [8-10]. It has been reported that SPD has anti-inflammatory and antioxidant abilities, which plays a important role in the protection of cells from free radical-mediated damage [11]. Mutations in the SPD synthase gene produced more apparent ROS damage to fibroblast cells than normal cells [12]. SPD is a potential candidate antioxidant, which may be independently involved in the protective mechanism against ROSmediated damage $[12,13]$. Notably, SPD with antioxidant and anti-inflammatory effects was significantly increased in cartilage and synovial fluid of patients with osteo-, rheumatoid- and post-traumatic arthritis [13]. These findings suggested the clinical significance of the antioxidant and anti-inflammatory effects of SPD for OA therapy, although it has little application. SPD is susceptible to enzymatic or chemical 
degradation in the joint cavity, which requires repeated intra-articular injection, limiting their bioavailability [14-17]. To improve their efficacy, suitable carriers for SPD is of significance [18, 19].

PAN is a bio-compatible polymer widely used in tissue engineering with unique properties such as biocompatibility, non-hydrophobicity, strength and stretchability, resistance to chemicals, stability and recyclability, microbial resistance, compatibility with some polar materials and solubility [20-22]. Electrospun nanofibers based on PAN have been reported for various applications such as cell cultures, antibacterial, antioxidants, bio-coatings and tissue engineering [23, 24]. In addition, PAN is a suitable polymer to produce electrospun nanofibers which could combine with the natural antioxidants such as polyamines, lignin and quaternary ammonium $[25,2746]$.

In our study, we proposed to graft SPD onto PAN to form a novel and sustainable antioxidant nanofibrous bio-scaffold to sustainably and efficiently prevent ROS damage in the therapy of OA (Scheme 1). Specifically, copolymer PAN-SPD was synthesized via electrostatic attraction and Mannich reaction, followed by the formation of nanofibrous membranes through electrospinning. The mechanical property of nanofibers was optimized by adjusting the ratio of PAN and SPD. The effectiveness of nanofibers for $\mathrm{OA}$ treatment were evaluated in $\mathrm{H}_{2} \mathrm{O}_{2}$-induced rat chondrocytes and $\mathrm{OA}$ joint model. Vitamin $\mathrm{E}, \mathrm{a}$ commonly used clinical antioxidant, was also electrospun with PAN, which served as control. The purpose of our research is to provide a novel biological antioxidant scaffold that can exert a long-term effect at the site of the lesion and thus delay the progression of OA.

\section{Materials And Methods}

\section{Materials}

Polyacrylonitrile (Macklin, 25014-41-9), Spermidine (Sigma-Aldrich, 124-20-9), Vitamin E (Sigma-Aldrich, 1406-66-2), N,N-Dimethylformamide (Aladdin, D112004), 2,2'-azinobis(3-ethylbenzothiazoline-6-sulfonate) (Solarbio, IA0010), Potassiumbromide (Sigma-Aldrich, 7758-02-3), Chloroform-D (Adamas, 86549-6), $\mathrm{H}_{2} \mathrm{O}_{2}$ (Fisher Chemical, H325-500), ethylenediaminetetraacetic acid (EDTA, Sigma-Aldrich, EDS500G), NaCN (Alfa Aesar, L13278), 2,2 diphenyl-1-picryl hydrazyl (DPPH, Sigma-Aldrich, D9132), Sodium nitroferricyanide dihydrate (Aladdin, 13755-38-9), sodium nitroprusside (Sigma-Aldrich, PHR1423), HCl (baker analyzed, 0000170466)

\section{Synthesis of PAN-SPD electrospun nanofibers}

Briefly, $0.6 \mathrm{~g}$ of PAN was first added to $10 \mathrm{~mL} \mathrm{~N}, \mathrm{~N}$-dimethylformamide (DMF), then keep stirring at room temperature for $12 \mathrm{~h}$ to obtain a clear, pale yellow solution. Then, the $0.05 \mathrm{~g}, 0.1 \mathrm{~g}, 0.15 \mathrm{~g}$ of SPD and $\mathrm{NaOH}$ were added into the solution respectively and stirred $48 \mathrm{~h}$ at room temperature to obtain the precursor solution. After stirring at room temperature until the solutions were well dispersed, each group of polymer solutions was transferred to a $10 \mathrm{~mL}$ syringe with a 22-gauge nozzle. The push rate was set to $0.8 \mathrm{~mL} / \mathrm{h}$ and the voltage of the electric field was set to $15 \mathrm{kV}$. Then, the aluminum foil was adhered to the receiver about $15 \mathrm{~cm}$ from the nozzle, and the program was started and electrospun at $25{ }^{\circ} \mathrm{C}$ for 
about $12 \mathrm{~h}$. Then drying in a vacuum dryer $24 \mathrm{~h}$ at $25^{\circ} \mathrm{C}$, the electrospun nanofibers were collected on aluminum foil for the next experiments. The preparation of pure PAN and PAN-VE (Containing 20\% vitamin E) electrospun nanofibers were using the same conditions. The composition of the nanofibers was list in (Additional file 4:Table S1).

\section{Characterization of nanofibers}

The molecular structure of PAN-SPD copolymer was investigated by Fourier transform infrared spectroscopy (FTIR) (Spectrum 2000, Perkin Elmer, USA). The nanofibers were dried and a thin layer of gold ions was sputtered on their surface, and then the surface morphology and fiber diameter were observed by scanning electron microscopy (SEM) (JSM6700F, JEOL, Japan). At least 50 fiber diameters in the the field of vision were analyzed using Image $\mathrm{J}$, and then the average fiber diameter was calculated. The crystal structures of the components in the different groups of copolymers were characterized by diffraction of x-rays (XRD) (MAXima XRD-7000, SHIMADZU, Janpan).

\section{Mechanical testing}

For mechanical testing, each group of nanofibers (thickness $\sim 250 \mu \mathrm{m}$ ) were cut into rectangles of size 10 $\times 20 \mathrm{~mm}(\mathrm{n}=5)$. Tensile testing is carried out by means of a uniaxial tensile test module (Instron 5942, USA). The measurement length was set to $20 \mathrm{~mm}$ and the stretch rate was $10 \mathrm{~mm} / \mathrm{min}$. A total of five nanofibers of each group were tested.

\section{Assessing antioxidant activity}

The scavenging activities of free radical were evaluated by 2, 2'-azino-bis(3-ethylbenzothiazoline-6sulfonic acid) (ABTS) radical scavenging test (Aladdin Reagent, China) through the provided instructions [29]. PAN-SPD nanofibers reduces $\mathrm{H}_{2} \mathrm{O}_{2}$ to water: $2 \mathrm{H}_{2} \mathrm{O}_{2} \rightarrow \mathrm{O}_{2}+2 \mathrm{H}_{2} \mathrm{O}$. The $\mathrm{H}_{2} \mathrm{O}_{2}$-scavenging capacities of PAN-SPD nanofibers was assessed using a portable dissolvedoxygen meter (Rex, JPBJ-608, China) at room temperature. After automatic calibration and zeroing, $20 \mathrm{mg}$ of each fiber and $20 \mathrm{mg}$ free SPD and VE was soaked in $10 \mathrm{~mL}$ of neutral phosphate buffered saline (PBS) and $1 \mathrm{~mL} 30 \% \mathrm{H}_{2} \mathrm{O}_{2}$ was added into solution and measurement of the generated oxygen concentration using one probe. Besides, we test the catalytic stability of the PAN, PAN-SPD (0.1) and PAN-VE nanofibers. After 10 minutes of reaction with $\mathrm{H}_{2} \mathrm{O}_{2}$ under the above conditions, solution was centrifuged at $10000 \mathrm{rpm}$ for $5 \mathrm{~min}$, then removed supernatant and washed nanofiber three times using PBS. Then repeated the reaction in same condition for a total of five cycles and the catalytic stability rate was calculated by the oxygen concentration of each cycle compared to the first cycle. Then, the 1,1-diphenyl-2-octanohydrazide (DPPH) clearance test was performed to assess the capacity of each group of nanofibers to scavenge DPPH free radical. Firstly, each group of nanofibers $(20 \mathrm{mg})$ was immersed in $20 \mathrm{~mL}$ DPPH/Methanol solution and centrifuged at $10000 \mathrm{rpm} / \mathrm{min}$ for $3 \mathrm{~min}$ at each set time point, after which the supernatant was taken out to measure the absorbance at $517 \mathrm{~nm}$. The degree of inhibition of DPPH radicals was indicated by measuring the change in absorbance of the experimental group compared to the control group and the antioxidant activity was calculated according to the formula and the results were expressed as DPPH inhibition (\%). 
The free radical scavenging activity of each group of nanofibers was further evaluated by superoxide anion scavenging ability test kit (Beyotime Biotechnology), hydroxyl radical scavenging ability test kit (Beyotime Biotechnology). The activity of the nanofibers in scavenging superoxide anions and hydroxyl radicals was assessed by measuring absorbance values at $530 \mathrm{~nm}$ and $536 \mathrm{~nm}$ after 0.5 and $1 \mathrm{~h}$, respectively. The nitric oxide scavenging activities of PAN-SPD nanofibers were tested by measuring the inhibition of the generation of nitrite. Nitric oxide was generated by sodium nitroprusside (SNP) (Aladdin Reagent, China) in an aqueous solution at physiological $\mathrm{pH}$ and was then detected by the Griess reagent after reaction with dissolved oxygen to form nitrite. Scavengers of NO compete with oxygen, leading to reduced production of $\mathrm{NO}_{2}{ }^{-} \cdot 1 \mathrm{~mL}$ PBS $(\mathrm{pH} 7.4,20 \mathrm{mM})$ of SNP $(10 \mathrm{mM})$ and PAN-SPD nanofibers $(20 \mathrm{mg})$ were incubated for $150 \mathrm{~min}$ at $25^{\circ} \mathrm{C}$. Subsequently, centrifuged the working solution at $12000 \mathrm{rpm}$ for 10 min, and the concentration of $\mathrm{NO}_{2}{ }^{-}$was then assessed by Nitric Oxide Assay Kit (Beyotime Biotechnology) and the OD540 was recorded.

\section{The degradation of PAN-SPD nanofibers}

For the assessment of degradation rates, the groups of nanofibers were soaked in PBS at $37^{\circ} \mathrm{C}$, physiological pH conditions for 8 weeks. In first week the each group of nanofibers in EP tubes were freezed dried and then weighted every day. In 2-8 weeks, each group of nanofibers were freeze drying and then weighted every 7 days. The rate of degradation is calculated by the weight loss over the initial weight.

\section{Cell culture}

Articular cartilage was harvested from 3-5 day old Sprague-Dawley (SD) suckling rat, and primary chondrocytes were isolated, digested and extracted. (Experimental Animal Center of Guangxi Medical University). The articular cartilage was finely cut with tissue scissors and wished 2-3 times using sterile PBS. After that, the cartilage was first soaked in $0.25 \%$ Trypsin-EDTA Solution (Solarbio, China) for 30 min, then incubated with $2 \mathrm{mg} / \mathrm{mL}$ of collagenase II (Solarbio, China) for 4-6 $\mathrm{h}$ in incubator with condition of $37^{\circ} \mathrm{C}$ and $5 \% \mathrm{CO}_{2}$. Then the supernatant was centrifuged at $1000 \mathrm{rpm} / \mathrm{min}$ for $5 \mathrm{~min}$ and added to Dulbecco's modified Eagle medium (DMEM) for incubation, and the remaining precipitate was continued to be digested overnight. The primary chondrocytes were cultured in DMEM (Gibco, USA) containing 10\% fetal bovine serum (Gibco, USA) and 1\% penicillin/streptomycin (Solarbio, China) in an incubator with condition of $37^{\circ} \mathrm{C}$ and $5 \% \mathrm{CO}_{2}$. After the primary cells reached more than $90 \%$ fusion, using $0.25 \%$ Trypsin-EDTA Solution to digested chondrocytes and passaged in the ratio of 1:3. We chosen the P2 generation chondrocytes for next experiments.

\section{Cell adhesion assay}

Chondrocytes were seeded on each group of nanofiber scaffolds and cultured in an incubator for 3-5 days. Then fixed the chondrocytes with $4 \%$ glutaraldehyde at $4{ }^{\circ} \mathrm{C}$ overnight and the following day were dehydrated with $50 \%, 70 \%, 90 \%$, and $100 \%$ ethanol sequentially and dehydration in hexamethyldisilane 
under vacuum for $1 \mathrm{~h}$. After the Au icon sputter coating of the nanofibers, the scaffolds for cell seeding were observed by SEM.

\section{$\mathrm{H}_{2} \mathrm{O}_{2}$-induced chondrocytes and treatment}

The groups were designated as (1) Control, (2) $\mathrm{H}_{2} \mathrm{O}_{2}$, (3) PAN $+\mathrm{H}_{2} \mathrm{O}_{2}$, (4) PAN-SPD $+\mathrm{H}_{2} \mathrm{O}_{2}$, (5) PAN$\mathrm{VE}+\mathrm{H}_{2} \mathrm{O}_{2}$. First, each group of nanofibers was cut to the size of a 12-well cell culture plate. Then, the nanofibers were immersed in $75 \%$ ethanol for $2 \mathrm{~h}$ to sterilization and washed 2-3 times with sterile PBS to wash out the residual ethanol. After that, the nanofibers were sterilized by exposed to UV light for at least 8 h. Approximately $15 \times 10^{4} \mathrm{P} 2$ generation chondrocytes were seeded on PAN, PAN-SPD (0.05), PAN-SPD (0.1), PAN-SPD (0.15) and PAN-VE nanofibers with $0.4 \mathrm{mmol} / \mathrm{L} \mathrm{H}_{2} \mathrm{O}_{2}$ for $24 \mathrm{~h}$. Based on the results, we selected PAN-SPD (0.1) nanofibers with the highest cellular activity and antioxidant activity for the next experiments.

\section{Cell viability assay}

The cell viability on PAN, PAN-SPD (0.05), PAN-SPD (0.1), PAN-SPD (0.15) and PAN-VE nanofibers were assayed by Cell Counting Kit-8 (CCK-8) test (Beyotime Biotechnology).

\section{Assessing of SOD, CAT and GSH level in chondrocytes}

According to the manufacturer's instructions, the antioxidant enzyme activity in chondrocytes of different treatment groups were measured using SOD, CAT (Solarbio, China) and GSH activity assay kits (Beyotime Biotechnology) respectively.

\section{Assay of ROS levels in vitro}

Intracellular ROS levels in different groups were analyzed using a reactive oxygen species detection kit (Beyotime Biotechnology). After induced with $400 \mu \mathrm{M} \mathrm{H}_{2} \mathrm{O}_{2}$ for $24 \mathrm{~h}$, chondrocytes on PAN, PAN-SPD (0.1), and PAN-VE nanofibers were incubated with $1 \mu \mathrm{M} 2$ ',7'-Dichlorodihydrofluorescein diacetate (DCFH-DA) under the condition of $37^{\circ} \mathrm{C}, 5 \% \mathrm{CO}_{2}$ for $30 \mathrm{~min}$ in the dark. After rinsed by PBS 2-3 times, flow cytometry was used to measure ROS in chondrocytes (ex/em $488 \mathrm{~nm}$ and $525 \mathrm{~nm}$ ).

\section{RNA extraction and qRT-PCR}

The mRNA expression levels of pro-inflammatory factors including MMP-13, IL-6, TNF-a, iNOS, COX-2 and cartilage-related factors Col2a1 in cells of different treatment groups were analyzed by qRT-PCR. Firstly, we used HiPure Total RNA Mini Kit (Magen, China) to extracted the total RNA from chondrocytes in different groups. Subsequently, the RNA transcription and qRT-PCR were performed as described in previous studies [26]. The designed primers were listed in (Additional file 5: Table S2). The mRNA expression levels were calculated using the $2^{-\Delta \Delta C t}$ method and GADPH was used as an internal reference gene. Each gene was analyzed in triplicate under the same conditions to reduce randomization error. 


\section{OA rat model establish}

8-week-old male SD rats weighing $250 \pm 20 \mathrm{~g}$ were used to assess the effect of nanofibers in the treatment of $\mathrm{OA}$ in vivo. All animal experiments were performed in accordance with the guidelines of the Animal Research Ethics Committee of Guangxi Medical University (Protocol Number: 2020-10-27) and all animal operations were conducted strictly according to the National Institutions of Health Guide for the Care and Use of Laboratory Animals. To established a model of OA in SD rats, we used surgical induction combined with exercise loaded method. After anesthetizing the rats with $3 \%$ pentobarbital sodium, anterior cruciate ligament transection (ACLT) was performed on the right and left knee joints of the rats. At the same time, the same skin incisions were made on the right and left knee joints of the rats as a sham-operated group. After surgery, rats were forced to exercise for more than $2 \mathrm{~h}$ per day.

\section{In vivo osteoarthritis treatment of PAN-SPD nanofibers}

After exposing the joint cavity and severing the anterior cruciate ligament, different groups of nanofibers were completely covered on the joint surface. The rats were divided into 5 groups: (1) sham group, (2) OA group: ACLT-operated with no treatment, (3) PAN group: OA treated with PAN nanofibers, (4) PAN-SPD group: OA treated with PAN-SPD (0.1) nanofibers, and (5) PAN-VE group: OA treated with PAN-VE nanofibers. At 4 and 8 weeks after ACLT surgery, rats in each group were executed with $3 \%$ sodium pentobarbital overdose anesthesia and the knee joints were harvested for the next experiments.

\section{Macroscopic observation}

After harvesting the knee joint of the rat, excess muscle and soft tissue were removed. Then, macroscopic observations were done and images of the tibial and femoral surfaces of the knee joint were obtained.

\section{Histological evaluation}

For the purpose of histological assessment, hematoxylin and eosin (H\&E) and safranin-O staining was used. In general, the cartilage was fixed in $4 \%$ paraformaldehyde for $48 \mathrm{~h}$ and then decalcified in EDTA decalcification solution (Boster, China) in an ultrasound machine for 4 weeks. Finally, the cartilage tissue

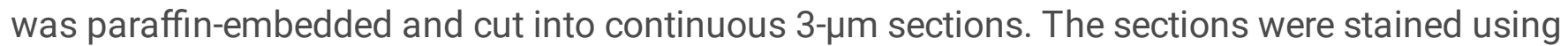
Hematoxylin and Eosin (H\&E) (Solarbio, China) and Safranin-O (Solarbio, China) according to the instructions of the kit. Observation and acquisition of section staining images using a microscope (Olympus, Japan).

\section{Immunohistochemical staining}

The protein expression levels of MMP-13 and IL-6 in articular cartilage were assessed by immunohistochemical staining. Briefly, after dewaxing the sections with xylene, the sections were incubated with the primary antibody MMP-13 (PROTEINTECH, USA, 1:200) and IL-6 (PROTEINTECH, USA, 1:200) overnight at $4^{\circ} \mathrm{C}$ in the dark. After washed 2-3 times by PBS, the sections were incubated with the second antibody (ZSGB-Bio, China) for 15 minutes and biotin-labeled Horseradish Peroxidase (ZSGB-Bio, 
China) for 15 minutes. The sections were then stained with 3,3-diaminobenzidine tetrahydrochloride (DAB) kit (ZSGB-Bio, China) according to the kit instructions, after that the sections were stained with hematoxylin for $5 \mathrm{~min}$. Then, the sections were sealed with neutral resin for observed and obtained pictures by microscope (Olympus, Japan).

\section{Statistical analysis}

All results are presented as mean \pm SD or median (scatter plot), and statistical analysis was performed using one-way analysis of variance (ANOVA) and, if differences were significant, the least significant difference (LSD) test. $\mathrm{P}<0.05$ indicates that the results are statistically significant. All experiments were repeated three times under the same conditions.

\section{Results}

\section{PAN-SPD nanofibers synthesis and characterizations}

The macroscopic observation and the surface morphology of different nanofibers were shown in (Fig. 1a, b and Additional file 1: Fig. S1). The fibers are uniform, smooth and randomly distributed. The neat PAN has an avarage fiber diameter of $288 \pm 20.3 \mathrm{~nm}$, while fibers electrospun from PAN-SPD copolymers were presented with much larger size which increased with content of SPD. PAN-VE has the fibre size close to PAN-SPD (0.05). To evaluate the mechanical properties of the nanofibers, stress-strain tests were performed. As shown in Fig. 1c, tensile tests demonstrated that tensile strength increased in the order of PAN, PAN-VE and PAN-SPDs. For PAN-SPDs, tensile stress increased with the content of SPD. In addition, the trend of Young's modulus of PAN, PAN-VE and PAN-SPDs were greater than PAN, and that of the PANSPD (0.1) was the largest (Fig. 1d). The results indicated that PAN-SPD (0.1) nanofibers have the most excellent mechanical properties.

The X-ray diffraction (XRD) pattern of PAN, PAN-VE and PAN-SPD (Fig. 1e) show that the two peaks (17 and $30^{\circ}$ ) were related to the hexagonal lattice structure of PAN [28], and the peak (34 $)$ of PAN-SPD is belong to SPD [50]. The results indicated that the PAN-SPD was successfully synthesized. Then, the chemical bonds of each nanofibers were identified by FTIR. It can be seen that, all of the samples had the absorption peaks at $2248 \mathrm{~cm}^{-1}$ due to the stretching vibration of $\mathrm{C} \equiv \mathrm{N}$ groups which is the characteristic bands of PAN chain $[52,53]$. In addition, the curve of PAN-SPD shown the peaks at $3279 \mathrm{~cm}^{-1}$ corresponding to $-\mathrm{NH}_{2}$ stretching vibration, and the peak at $1138 \mathrm{~cm}^{-1}$ corresponding to $\mathrm{C}=0$ stretching vibration which indicated that SPD has been successfully grafted to PAN [54] (Fig. 1f). When tested in PBS at $37^{\circ} \mathrm{C}$ (Fig. 1 $\mathbf{g}$ ), the degradation rates of each nanofibers increased rapidly during the first 6 to 7 days, then gradually stabilized until 56 days, thus confirming the biodegradable and biostability of the nanofibers.

Antioxidant activity of the PAN-SPD nanofibers 
The results of the ABTS assay were shown in Fig. 2a. The PAN membranes had low radical scavenging activity. However, the PAN-SPDs nanofibers exhibited higher dose-dependent radical scavenging activity. And more than $50 \%$ of ABTS radical were eliminated in PAN-SPD (0.15), while the PAN-VE only displayed $27 \%$ scavenging activity, indicating that SPD is the main component of nanofibers that can exert antioxidant activity. As shown in (Additional file 2: Fig. S2), the DPPH Inhibition rate of PAN nanofibers was only $14 \%$, due to spontaneous decomposition [31]. Compared with PAN and PAN-VE, PAN-SPDs displays the more effective inhibition of DPPH. At the same time, we also can prove their antioxidant activity by the amount of $\mathrm{O}_{2}$ generated following the decomposition of $\mathrm{H}_{2} \mathrm{O}_{2}$ (Fig. 2b). The PAN-SPDs decompose $\mathrm{H}_{2} \mathrm{O}_{2}$ effectively in a concentration-dependent manner, and the yield of decomposed $\mathrm{O}_{2}$ was much higher than that of PAN and PAN-VE. Moreover, PAN-SPD, PAN and PAN-VE nanofibers showed nearly the same catalytic activity as the original solution with the catalytic stabilization rates of all above 95\% after 5 cycles reaction(Fig. $2 \mathrm{c}$ and Additional file 3: Fig. S3). And the PAN-SPD (0.1) nanofiber showed the highest catalytic activity in all the groups, indicating that the PAN-SPD (0.1) can sustainably and efficiently scavenged $\mathrm{H}_{2} \mathrm{O}_{2}$. We further evaluated the radical scavenging activity of each nanofibers using superoxide anion assay, hydroxyl radical assay and nitric oxide assay kits. As shown in Fig. 2d-f, PAN-SPD (0.15) nanofiber showed $44.39 \%$ inhibition of superoxide anion after 30 mins, $31.99 \%$ inhibition of hydroxyl radical after 60 mins and $42.85 \%$ inhibition of nitric oxide after 150 mins, respectively, that were all much higher than PAN and PAN-VE.

\section{PAN-SPD nanofibers for the treatment of osteoarthritis in vitro}

To evaluate cell adhesion, chondrocytes-seeded nanofibers were viewed using SEM to examined cell spreading. As shown in Fig. 3a, chondrocytes can adhere well to all nanofibers, which is conducive to cell proliferation and diffusion.

The activity of chondrocytes seeded on all nanofibers was evaluated with the CCK-8 assay. As shown in Fig. $3 b$, the cell survival rates of all nanofibers were above $85 \%$, indicating that they have better biocompatibility [32]. Especially, the PAN-SPD (0.1) nanofiber exhibited the highest cellular activity (94.73\%). Similarly, after $\mathrm{H}_{2} \mathrm{O}_{2}$ treatment to induce oxidative stress injury on chondrocytes (Fig. 3C), PANSPD (0.1) owned the highest cell viability (83.19\%), demonstrating it is the most effective to resist the oxidative stress damage induced by $\mathrm{H}_{2} \mathrm{O}_{2}$ to protect chondrocytes. Therefore, we selected PAN-SPD (0.1) nanofiber for further biological research.

We assessed the levels of antioxidant factors such as SOD, CAT and GSH in different groups under the stimulation of $\mathrm{H}_{2} \mathrm{O}_{2}$. As shown in Fig. 3d-f, SOD, CAT activity and GSH levels were significantly downregulated in cells treated with $\mathrm{H}_{2} \mathrm{O}_{2}$ and $\mathrm{PAN}+\mathrm{H}_{2} \mathrm{O}_{2}$. In comparison, the PAN-SPD (0.1) $+\mathrm{H}_{2} \mathrm{O}_{2}$ significantly increased the activities of SOD, CAT and GSH levels which weresiginificantly higher than PAN-VE+ $\mathrm{H}_{2} \mathrm{O}_{2}$. Furthermore, we used the 2', 7'-dichlorodiacetate (DCFH-DA) and flow cytometry test to further study the ability of PAN-SPD (0.1) to eliminate intracellular ROS. The results of the flow cytometry (Fig. $\mathbf{3 g}$ ) shown that only $0.03 \%$ cells on PAN-SPD $(0.1)$ nanofibers were positive by DCF fluorescence, which was much 
less than $\mathrm{H}_{2} \mathrm{O}_{2}$ (77.92\%), PAN-VE+ $\mathrm{H}_{2} \mathrm{O}_{2}(18.86 \%)$ and $\mathrm{PAN}+\mathrm{H}_{2} \mathrm{O}_{2}(44.52 \%)$. These results demonstrated that PAN-SPD (0.1) nanofibers can effectively scavenge intracellular ROS to attenuate $\mathrm{H}_{2} \mathrm{O}_{2}$-mediated oxidative stress damage.

To further explore the anti-inflammatory effect of the PAN-SPD nanofibers, we used quantitative qPCR analysis to evaluate MMP-13, TNF-a, IL-6, COX-2, iNOS and Col2a1 expressions of chondrocytes stimulated with $\mathrm{H}_{2} \mathrm{O}_{2}$. As shown in Fig. 4a-f, compared with the control group, the mRNA expression levels of inflammatory factors (IL-6, TNF-a, iNOS, COX-2) and cartilage-degrading factor (MMP-13) was significantly increased after $\mathrm{H}_{2} \mathrm{O}_{2}$ stimulation, except that the cartilage specific Col2a1 expression was decreased. Compared with $\mathrm{H}_{2} \mathrm{O}_{2}$ and $\mathrm{PAN}+\mathrm{H}_{2} \mathrm{O}_{2}$, PAN-SPD (0.1) $+\mathrm{H}_{2} \mathrm{O}_{2}$ treatment down-regulated the increased of the IL-6, TNF- $a$, MMP-13 and iNOS. For the TNF- $a$, that of the PAN-SPD (0.1)+ $\mathrm{H}_{2} \mathrm{O}_{2}$ was lower than PAN-VE+ $\mathrm{H}_{2} \mathrm{O}_{2}$. Compared with $\mathrm{H}_{2} \mathrm{O}_{2}$, the levels of COX-2 for the PAN-SPD (0.1) $+\mathrm{H}_{2} \mathrm{O}_{2}$ was decreased, while the levels of Col2a1 was increased. Therefore, PAN-SPD $(0.1)+\mathrm{H}_{2} \mathrm{O}_{2}$ has a better anti-inflammatory effect.

\section{PAN-SPD nanofibers attenuate OA progression in vivo}

After ACLT surgery and implanted PAN, PAN-VE and PAN-SPD (0.1) nanofibers to treated OA rat for 4 and 8 weeks (Fig. 5a). As assessed macroscopically in the OA and PAN nanofibers group, the joint surfaces were very rough and defective. After treated by PAN-VE nanofibers, the cartilage lesions were slightly improved, although still destructive. However, the articular cartilage lesions were significantly reduced in the group receiving PAN-SPD (0.1) nanofiber treatment, the joint surfaces seem smooth and shiny, which was similar to normal cartilage .

The results of the OA and PAN group from HE staining showed that the surface of chondrocytes became swollen and rounded, with the chondrocyte distribution disordered and irregular. And cracks and defects in the articular surface were also observed. In contrast, in the PAN-SPD (0.1) group, the shape and distribution of chondrocytes were more regular, and the cartilage surfaces were more smooth and intact, which was better than PAN-VE group (Fig. 5b). In addition, in the OA and PAN group, we can observed that staining of the cartilage layer of safranin $O$ reduced, the joint space narrowed, cartilage surfaces defected. However, after treatment with PAN-SPD nanofibers, cartilage layer thickness and integrity were significantly better, which was superior to the PAN-VE group (Fig. 6a).

As a marker of proinflammatory and cartilage-degrading, MMP-13 and IL- 6 are commonly used in the evaluation of OA [51]. According to immunohistochemistry staining (Fig. 6b, c), the OA and PAN group secreted large amounts of MMP-13 and IL-6. The positive staining of MMP-13 and IL- 6 in PAN-VE nanofibers group was still obvious. However, these were rescued by PAN-SPD (0.1) nanofiber treatments. All these results indicated that PAN-SPD (0.1) nanofibers could downregulate MMP-13 and IL-6 inflammatory factors, and prevent the development of $\mathrm{OA}$, consistent with previous conclusions.

\section{Discussion}


Previous studies have shown that ROS lead to chondrocyte apoptosis and cartilage degeneration which accelerate OA progression [33-35]. SPD could act as free radical scavengers, but it would be rapidly cleared from the joint cavity $[13,36]$. With the development of tissue engineering technology, bioscaffolds have been used as carriers to prolong the retention time of antioxidants in the joint cavity [3739]. In this study, we report a novel PAN-SPD nanofibers, and investigated the effectiveness in the treatment of $O A$.

The PAN-SPD nanofiber membranes were fabricated by charge mutual attraction and then the Mannich reaction by reacting the methylene group of PAN with the amino group of SPD. After adjusting the ratio of SPD/PAN for an optimization, the produced nanofibers show dense and randomly oriented fibers (Fig. 1a) and have excellent tensile resistance strength (Fig. 1c d). The mechanical properties of PAN-SPD nanofibers are nearly $4 \mathrm{MPa}$ higher than those of conventional PCL or PLGA nanofibers [20,30]. The Pure PAN nanofibers have almost no antioxidant capacity, but after grafting with SPD, PAN-SPD possess the antioxidant capacity, which is better than that of vitamin E (Fig. 2). Meanwhile, the resuls of catalytic stability test indicated that PAN-SPD nanofibers also have the ability to sustainable catalyzed (Fig. 2c and Fig. S3). Besides, PAN-SPD nanofibers also have more high and sustained antioxidant capacity than free SPD [36]. Interestingly, the mechanical property also increases with the increase of SPD amount, this may be due to the enhanced inter-molecular interaction forces between PAN and SPD(Fig. 1c, d). Both PAN and SPD have favorable bio-compatibility $[8,24]$, which is consistent with our results that PAN-SPD nanofiber membranes are also biocompatible. The cell viability on pure PAN nanofibers was approximately $93.78 \%$ and did not change significantly after the addition of SPD and even higher on PANSPD (0.1) nanofibers with the cell viability of $94.73 \%$ (Fig. 3b). In OA joints, excessive oxidative stress damage is the main cause of chondrocyte damage and up-regulation of inflammatory factors [40, 41]. And our in vitro results showed that PAN-SPD nanofibers with moderate SPD content could increase $\mathrm{H}_{2} \mathrm{O}_{2}$-decreased cell viability to $83.19 \%$ (Fig. 3c). Further, we confirmed that PAN-SPD has the ability to scavenge intracellular ROS and down-regulating inflammatory factors such as MMP-13, IL-6, TNF-a, iNOS, and COX-2 while up-regulating antioxidant enzyme activities (SOD, CAT, GSH), indicating it restored ROS-mediated damage (Fig. 3d-g and Fig. 4).

Many common antioxidants such as polysaccharides, vitamin $\mathrm{E}$, and vitamin $\mathrm{C}$ have been used to treat OA [42-45]. However, traditional oral or intra-articular injections require repepetive administration [4648]. Meanwhile, according to previous studies, the effect of conventional antioxidants in the treatment of OA was not ideal [49]. Comparatively, our nanofibre membranes can avoid repepetive injection since they may supply sustained antioxidants accompanied with concomitant slow degradation. And the results of macroscopic observations and histological evaluation results showed that there was a substantial improvement in the articular cartilage lesions by PAN-SPD therapy, even approaching the sham group (Fig. 5 and Fig. 6). Moreover, pure PAN has little effect on alleviation of OA progression, which was close to OA group, indicating that SPD in PAN-SPD nanofibers plays the major role for OA therapy. Overall, intraarticular implantation of PAN-SPD nanofibers is a safe and effective treatment methods for OA. 
Therefore, we believe that PAN-SPD nanofiber membranes can serve as a good antioxidant bio-scaffold and a new synergistic, safe and long-lasting approach for the treatment of OA.

\section{Conclusion}

In conclusion, we synthesized a novel electrospun PAN-SPD nanofiber membranes with sustainable release of SPD as ROS scavenger for OA therapy, in an attempt to prevent rapid clearance and unstability of antioxidants in the joint cavity. PAN-SPD membranes showed dense and randomly oriented fibers that are suitable for cell growth. As novel ROS scavengers, PAN-SPD nanofibers can sustainably and effectively scavenge excessive ROS in $\mathrm{H}_{2} \mathrm{O}_{2}$ induced chondrocytes, activating antioxidant enzymes and down-regulating the expression of inflammatory factors, thus alleviating the OA progression in OA joints. This delayed the pathological process of chondrocytes death, cartilage destruction and degeneration. As a novel therapeutic measure, PAN-SPD nanofibers overcome the short-period retention, high dosage and poor efficacy of traditional OA treatment methods. Overall, this study provides a new electrospun nanofiber membrane for the treatment of OA through scavenging ROS.

\section{Declarations}

\section{Acknowledgments}

This study was financially supported by the National Natural Science Foundation of China (82160429), National key R\&D program of China (2018YFC1105900), the Guangxi Key Research and Development Plan (Grant No. GuikeAD19254003), Distinguished Young Scholars Program of Guangxi Medical University.

\section{Authors' contributionst}

SZG, BCL, MLH, LZ and JPZ designed experiments and wrote the manuscript. SZG, BCL and YL synthesized and analyzed the nanofibers. SZG, BCL, GHL and YLL conducted the vitro experiments and histopathological analysis. SZG, BCL and $Y X$ conducted the animal studies and subsequent analysis. SZG and BCL conducted the WB experiment. JPZ, BCL and YL helped to edit the figures. SZG, BCL, YLL, $\mathrm{GHL}, \mathrm{YX}$ performed statistical analysis of the data. All authors read and approved the final manuscript.

\section{Availability of data and materials}

The datasets used and/or analyzed during the current study are available from the corresponding author on reasonable request.

\section{Ethics approval and consent to participate}

Animal care was in accordance with institutional guidelines. All animal studies were performed in accordance with the ethical approval of the Institutional Ethics Committee of Guangxi Medical University. 


\section{Consent for publication}

Not applicable.

\section{Competing interests}

The authors declare no conflict of interest.

\section{Author details}

${ }^{a}$ Guangxi Engineering Center in Biomedical Material for Tissue and Organ Regeneration, The First Afliated Hospital of Guangxi Medical University, Nanning, Guangxi 530021, China. ${ }^{b}$ The Collaborative Innovation Center of Guangxi Biological Medicine, The First Afliated Hospital of Guangxi Medical University, Nanning, Guangxi 530021, China. Guangxi University of Chinese Medicine, Nanning, Guangxi 530200, China. ${ }^{\circ}$ Guangxi University of Chinese Medicine, Nanning, Guangxi 530200, China. ${ }^{1}$ Shangzhi Gao and Bingcai Long contributed equally to this work.

\section{References}

1. Mahmoudian A, Lohmander LS, Mobasheri A, Englund M, Luyten FP. Early-stage symptomatic osteoarthritis of the knee - time for action. Nat Rev Rheumatol. 2021;17(10):621-32.

2. Phillips RE. Review of Hip and Knee Osteoarthritis. JAMA. 2021;325(24):2504-5.

3. Wan WL, Lin YJ, Shih PC, Bow YR, Cui Q, Chang Y, Chia WT, Sung HW. An In Situ Depot for Continuous Evolution of Gaseous H2 Mediated by a Magnesium Passivation/Activation Cycle for Treating Osteoarthritis. Angew Chem Int Ed Engl. 2018;57(31):9875-9.

4. Lan Q, Lu R, Chen H, Pang Y, Xiong F, Shen C, Qin Z, Zheng L, Xu G, Zhao J. MMP-13 enzyme and pH responsive theranostic nanoplatform for osteoarthritis. J Nanobiotechnology. 2020;18(1):117.

5. Lepetsos P, Papavassiliou AG. ROS/oxidative stress signaling in osteoarthritis. Biochim Biophys Acta. 2016;1862(4):576-91.

6. Ning Y, Huo Y, Xue H, Du Y, Yao Y, Sedgwick AC, Lin H, Li C, Jiang SD, Wang BW, Gao S, Kang L, Sessler JL, Zhang JL. Tri-Manganese(III) Salen-Based Cryptands: A Metal Cooperative Antioxidant Strategy that Overcomes Ischemic Stroke Damage In Vivo. J Am Chem Soc. 2020;142(22):1021927.

7. Xiong W, Lan Q, Liang X, Zhao J, Huang H, Zhan Y, Qin Z, Jiang X, Zheng L. Cartilage-targeting poly(ethylene glycol) (PEG)-formononetin (FMN) nanodrug for the treatment of osteoarthritis. $J$ Nanobiotechnology. 2021;19(1):197.

8. Pegg AE. Functions of Polyamines in Mammals. J Biol Chem. 2016;291(29):14904-12.

9. Madeo F, Eisenberg T, Pietrocola F, Kroemer G. Spermidine in health and disease. Science. 2018;359(6374):eaan2788.

10. Green DR. Polyamines and Aging: A CLEAR Connection? Mol Cell. 2019;76(1):5-7. 
11. Carriche GM, Almeida L, Stüve P, Velasquez L, Dhillon-LaBrooy A, Roy U, Lindenberg M, Strowig T, Plaza-Sirvent C, Schmitz I, Lochner M, Simon AK, Sparwasser T. Regulating T-cell differentiation through the polyamine spermidine. J Allergy Clin Immunol. 2021;147(1):335-48.

12. Yuan X, Tian GG, Pei X, Hu X, Wu J. Spermidine induces cytoprotective autophagy of female germline stem cells in vitro and ameliorates aging caused by oxidative stress through upregulated sequestosome-1/p62 expression. Cell Biosci. 2021;11(1):107.

13. Adamo S, Cetrullo S, Guidotti S, Silvestri Y, Minguzzi M, Santi S, Cattini L, Filardo G, Flamigni F, Borzì RM. Spermidine rescues the deregulated autophagic response to oxidative stress of osteoarthritic chondrocytes. Free Radic Biol Med. 2020;153:159-72.

14. Sacitharan PK, Lwin S, Gharios GB, Edwards JR. Spermidine restores dysregulated autophagy and polyamine synthesis in aged and osteoarthritic chondrocytes via EP300. Exp Mol Med. 2018;50(9):1-10.

15. Yang L, Liu Y, Sun L, Zhao C, Chen G, Zhao Y. Biomass Microcapsules with Stem Cell Encapsulation for Bone Repair. Nanomicro Lett. 2021;14(1):4.

16. Bedingfield SK, Colazo JM, Di Francesco M, Yu F, Liu DD, Di Francesco V, Himmel LE, Gupta MK, Cho H, Hasty KA, Decuzzi P, Duvall CL. Top-Down Fabricated microPlates for Prolonged, Intra-articular Matrix Metalloproteinase 13 siRNA Nanocarrier Delivery to Reduce Post-traumatic Osteoarthritis. ACS Nano. 2021;15(9):14475-91.

17. Li X, Dai B, Guo J, Zheng L, Guo Q, Peng J, Xu J, Qin L. Nanoparticle-Cartilage Interaction: PathologyBased Intra-articular Drug Delivery for Osteoarthritis Therapy. Nanomicro Lett. 2021;13(1):149.

18. Kang LJ, Yoon J, Rho JG, Han HS, Lee S, Oh YS, Kim H, Kim E, Kim SJ, Lim YT, Park JH, Song WK, Yang S, Kim W. Self-assembled hyaluronic acid nanoparticles for osteoarthritis treatment. Biomaterials. 2021;275:120967.

19. Zhao C, Chen J, Ye J, Li Z, Su L, Wang J, Zhang Y, Chen J, Yang H, Shi J, Song J. Structural Transformative Antioxidants for Dual-Responsive Anti-Inflammatory Delivery and Photoacoustic Inflammation Imaging. Angew Chem Int Ed Engl. 2021;60(26):14458-66.

20. Chen S, Wang H, McCarthy A, Yan Z, Kim HJ, Carlson MA, Xia Y, Xie J. Three-Dimensional Objects Consisting of Hierarchically Assembled Nanofibers with Controlled Alignments for Regenerative Medicine. Nano Lett. 2019;19(3):2059-65.

21. Liu C, Wang Z, Yao X, Wang M, Huang Z, Li X. Sustained Biochemical Signaling and Contact Guidance by Electrospun Bicomponents as Promising Scaffolds for Nerve Tissue Regeneration. ACS Omega. 2021;6(48):33010-7.

22. Liang Y, Mitriashkin A, Lim TT, Goh JC. Conductive polypyrrole-encapsulated silk fibroin fibers for cardiac tissue engineering. Biomaterials. 2021;276:121008.

23. Shin J, Jeong S, Kim J, Choi YY, Choi J, Lee JG, Kim S, Kim M, Rho Y, Hong S, Choi JI, Grigoropoulos $\mathrm{CP}, \mathrm{Ko} \mathrm{SH}$. Dynamic Pore Modulation of Stretchable Electrospun Nanofiber Filter for Adaptive Machine Learned Respiratory Protection. ACS Nano. 2021;15(10):15730-40. 
24. Xiao YQ, Cao YJ, Xin BJ, Liu Y, Sun YG. Fabrication and characterization of electrospun cellulose/polyacrylonitrile nanofibers with Cu(II) ions. Cellulose. 2018;25(3):2955-63.

25. Dixita S, Palb S. Recent Advanced Technologies in the Processing of Hybrid Reinforced Polymers for Applications of Membranes. Polym Polym Compos. 2016;24(4):289-305.

26. Nakamura H, Shimamura S, Yasuda S, Kono M, Kono M, Fujieda Y, Kato M, Oku K, Bohgaki T, Shimizu T, Iwasaki N, Atsumi T. Ectopic RASGRP2 (CaIDAG-GEFI) expression in rheumatoid synovium contributes to the development of destructive arthritis. Ann Rheum Dis. 2018;77(12):1765-72.

27. Xue J, Xie J, Liu W, Xia Y. Electrospun Nanofibers: New Concepts, Materials, and Applications. Acc Chem Res. 2017;50(8):1976-87.

28. Youe WJ, Lee SM, Lee SS, Lee SH, Kim YS. Characterization of carbon nanofiber mats produced from electrospun lignin-g-polyacrylonitrile copolymer. Int J Biol Macromol. 2016;82:497-504.

29. Yang W, Shi X, Shi Y, Yao D, Chen S, Zhou X, Zhang B. Beyond the Roles in Biomimetic Chemistry: An Insight into the Intrinsic Catalytic Activity of an Enzyme for Tumor-Selective Phototheranostics. ACS Nano. 2018;12(12):12169-80.

30. Liang R, Zhao J, Li B, Cai P, Loh XJ, Xu C, Chen P, Kai D, Zheng L. Implantable and degradable antioxidant poly( $\varepsilon$-caprolactone)-lignin nanofiber membrane for effective osteoarthritis treatment. Biomaterials. 2020;230:119601.

31. Adhikari U, An X, Rijal N, Hopkins T, Khanal S, Chavez T, Tatu R, Sankar J, Little KJ, Hom DB, Bhattarai N, Pixley SK. Embedding magnesium metallic particles in polycaprolactone nanofiber mesh improves applicability for biomedical applications. Acta Biomater. 2019;98:215-34.

32. Ren K, Wang Y, Sun T, Yue W, Zhang H. Electrospun PCL/gelatin composite nanofiber structures for effective guided bone regeneration membranes. Mater Sci Eng C Mater Biol Appl. 2017;78:324-32.

33. Vaamonde-García C, Flórez-Fernández N, Torres MD, Lamas-Vázquez MJ, Blanco FJ, Domínguez H. Meijide-Faílde R. Study of fucoidans as natural biomolecules for therapeutical applications in osteoarthritis. Carbohydr Polym. 2021;258:117692.

34. Yang G, Fan M, Zhu J, Ling C, Wu L, Zhang X, Zhang M, Li J, Yao Q, Gu Z, Cai X. A multifunctional anti-inflammatory drug that can specifically target activated macrophages, massively deplete intracellular $\mathrm{H}_{2} \mathrm{O}_{2}$, and produce large amounts $\mathrm{CO}$ for a highly efficient treatment of osteoarthritis. Biomaterials. 2020;255:120155.

35. Xiong F, Qin Z, Chen H, Lan Q, Wang Z, Lan N, Yang Y, Zheng L, Zhao J, Kai D. pH-responsive and hyaluronic acid-functionalized metal-organic frameworks for therapy of osteoarthritis. J Nanobiotechnology. 2020;18(1):139.

36. Yuan H, Wu SX, Zhou YF, Peng F. Spermidine Inhibits Joints Inflammation and Macrophage Activation in Mice with Collagen-Induced Arthritis. J Inflamm Res. 2021;14:2713-21.

37. Deyle GD, Allen CS, Allison SC, Gill NW, Hando BR, Petersen EJ, Dusenberry DI, Rhon DI. Physical Therapy versus Glucocorticoid Injection for Osteoarthritis of the Knee. N Engl J Med. 2020;382(15):1420-9. 
38. Wei Y, Yan L, Luo L, Gui T, Jang B, Amirshaghaghi A, You T, Tsourkas A, Qin L, Cheng Z. Phospholipase A2 inhibitor-loaded micellar nanoparticles attenuate inflammation and mitigate osteoarthritis progression. Sci Adv. 2021;7(15):eabe6374.

39. Hou W, Ye C, Chen M, Gao W, Xie X, Wu J, Zhang K, Zhang W, Zheng Y, Cai X. Excavating bioactivities of nanozyme to remodel microenvironment for protecting chondrocytes and delaying osteoarthritis. Bioact Mater. 2021;6(8):2439-51.

40. Bolduc JA, Collins JA, Loeser RF. Reactive oxygen species, aging and articular cartilage homeostasis. Free Radic Biol Med. 2019;132:73-82.

41. Portal-Núñez S, Esbrit P, Alcaraz MJ, Largo R. Oxidative stress, autophagy, epigenetic changes and regulation by miRNAs as potential therapeutic targets in osteoarthritis. Biochem Pharmacol. 2016;108:1-10.

42. Grover AK, Samson SE. Benefits of antioxidant supplements for knee osteoarthritis: rationale and reality. Nutr J. 2016;15:1.

43. Chen Q, Shao X, Ling P, Liu F, Han G, Wang F. Recent advances in polysaccharides for osteoarthritis therapy. Eur J Med Chem. 2017;139:926-35.

44. Vinatier C, Merceron C, Guicheux J. Osteoarthritis: from pathogenic mechanisms and recent clinical developments to novel prospective therapeutic options. Drug Discov Today. 2016;21(12):1932-7.

45. Joshy KS, George A, Jose J, Kalarikkal N, Pothen LA, Thomas S. Novel dendritic structure of alginate hybrid nanoparticles for effective anti-viral drug delivery. Int J Biol Macromol. 2017;103:1265-75.

46. Chin KY, Ima-Nirwana S. The Role of Vitamin E in Preventing and Treating Osteoarthritis - A Review of the Current Evidence. Front Pharmacol. 2018;9:946.

47. Zhang W, Robertson WB, Zhao J, Chen W, Xu J. Emerging Trend in the Pharmacotherapy of Osteoarthritis. Front Endocrinol (Lausanne). 2019;10:431.

48. Hermann W, Lambova S, Muller-Ladner U. Current Treatment Options for Osteoarthritis. Curr Rheumatol Rev. 2018;14(2):108-16.

49. Bannuru RR, Osani MC, Vaysbrot EE, Arden NK, Bennell K, Bierma-Zeinstra SMA, Kraus VB, Lohmander LS, Abbott JH, Bhandari M, Blanco FJ, Espinosa R, Haugen IK, Lin J, Mandl LA, Moilanen E, Nakamura N, Snyder-Mackler L, Trojian T, Underwood M, McAlindon TE. OARSI guidelines for the non-surgical management of knee, hip, and polyarticular osteoarthritis. Osteoarthritis Cartilage. 2019;27(11):1578-89.

50. Zaccherini G, Aguilar F, Caraceni P, Clària J, Lozano JJ, Fenaille F, Castelli F, Junot C, Curto A, Formentin C, Weiss E, Bernardi M, Jalan R, Angeli P, Moreau R, Arroyo V. Assessing the role of amino acids in systemic inflammation and organ failure in patients with ACLF. J Hepatol. 2021;74(5):111731.

51. Latourte A, Cherifi C, Maillet J, Ea HK, Bouaziz W, Funck-Brentano T, Cohen-Solal M, Hay E, Richette P. Systemic inhibition of IL-6/Stat3 signalling protects against experimental osteoarthritis. Ann Rheum Dis. 2017;76(4):748-55. 
52. Farzin L, Sadjadi S, Shamsipur M, Sheibani S, Mousazadeh MH. Employing AgNPs doped amidoxime-modified polyacrylonitrile (PAN-oxime) nanofibers for target induced strand displacement-based electrochemical aptasensing of CA125 in ovarian cancer patients. Mater Sci Eng C Mater Biol Appl. 2019;97:679-87.

53. Geng Y, Zhang P, Wang Q, Liu Y, Pan K. Novel PAN/PVP Janus ultrafine fiber membrane and its application for biphasic drug release. J Mater Chem B. 2017;5(27):5390-6.

54. Ullah S, Hashmi M, Kharaghani D, Khan MQ, Saito Y, Yamamoto T, Lee J, Kim IS. Antibacterial properties of in situ and surface functionalized impregnation of silver sulfadiazine in polyacrylonitrile nanofiber mats. Int J Nanomedicine. 2019;14:2693-703.

\section{Supplemental Data}

Scheme 1 is available in the Supplementary Files section.

\section{Figures}




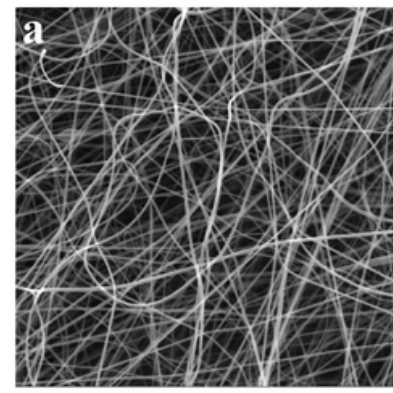

PAN

b

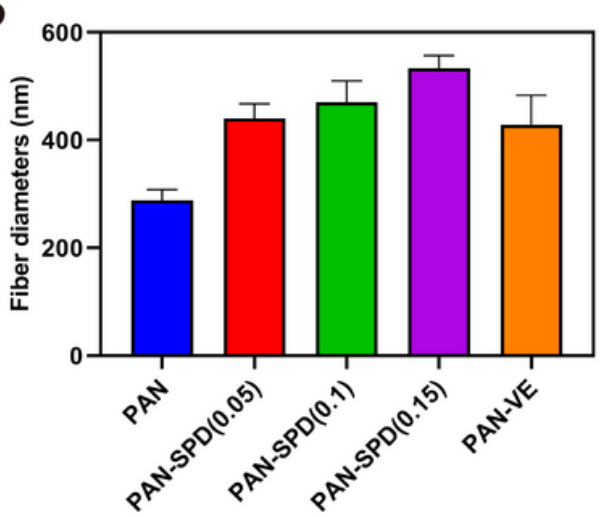

$\mathbf{e}$

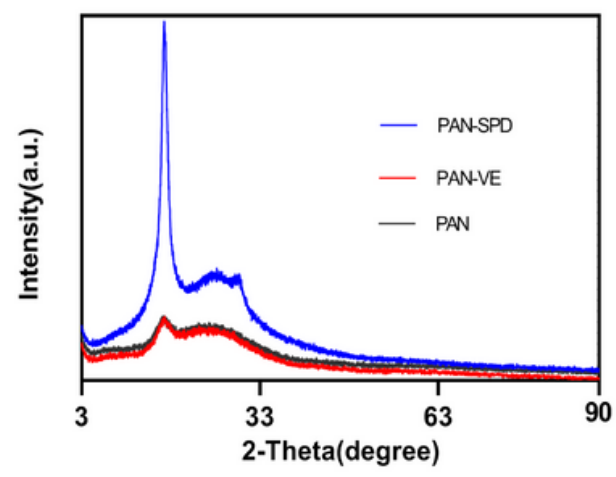

PAN-SPD (0.1)

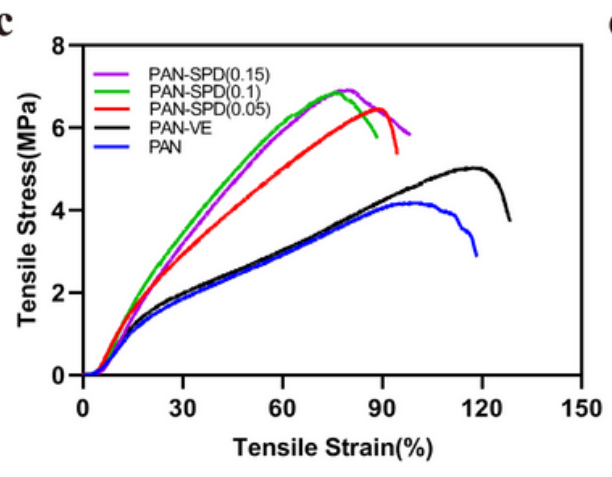

f

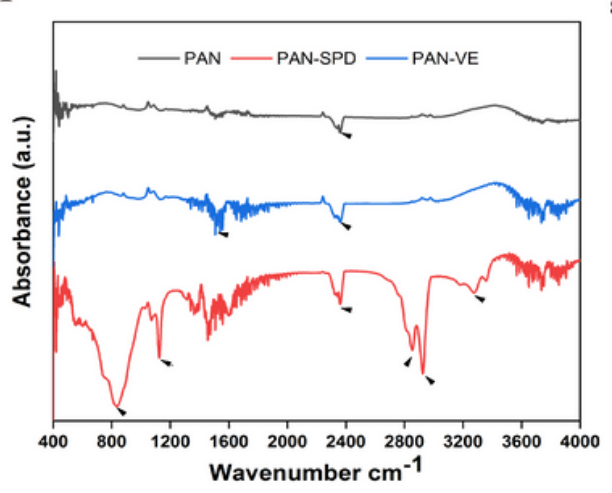

PAN-SPD (0.15)

d

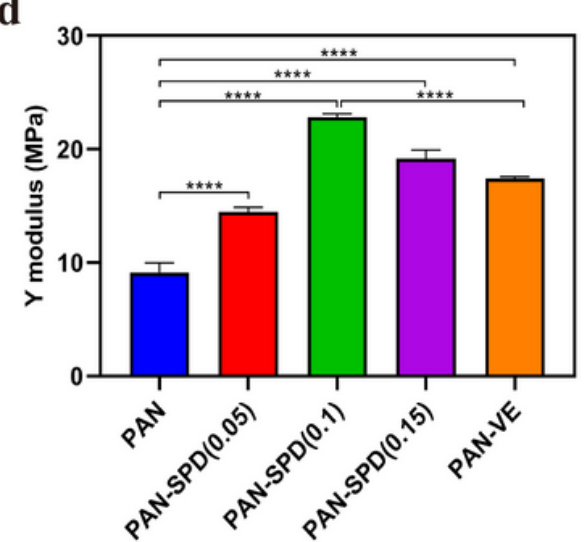

g

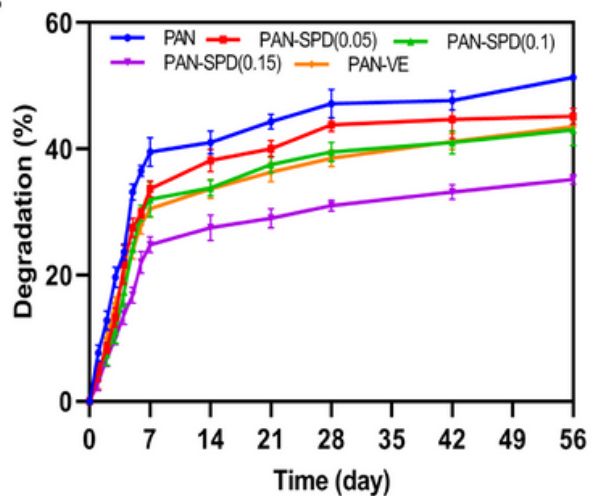

Figure 1

(a) SEM images of different nanofibers: PAN, PAN-SPD (0.05), PAN-SPD (0.1), PAN-SPD (0.15) and PANVE. The numbers indicate the weight (g) of SPD. (b) Fiber diameter. Data points represent mean \pm S.D. (n $=50$ ). (c) Tensile test (stress-strain curves) of the nanofibers. (d) Young's modulus. (e) XRD patterns of PAN, PAN-SPD (0.1) and PAN-VE. (f) FTIR spectrum of PAN, PAN-SPD (0.1) and PAN-VE. The characteristic peaks are indicated in the graph. (g) Degradation rate of different nanofibers: PAN, PANSPD (0.05), PAN-SPD (0.1), PAN-SPD (0.15) and PAN-VE. All data points represent mean \pm S.D. ${ }^{\star \star \star} \mathrm{P}<$ $0.001, * * P<0.01, * P<0.05$. 
a

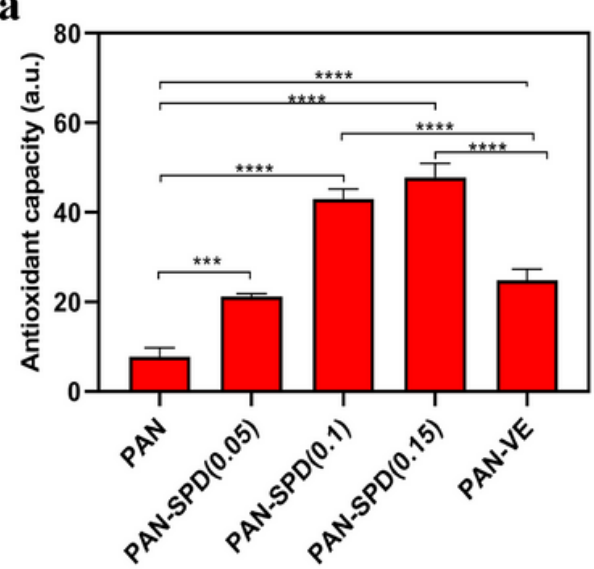

d

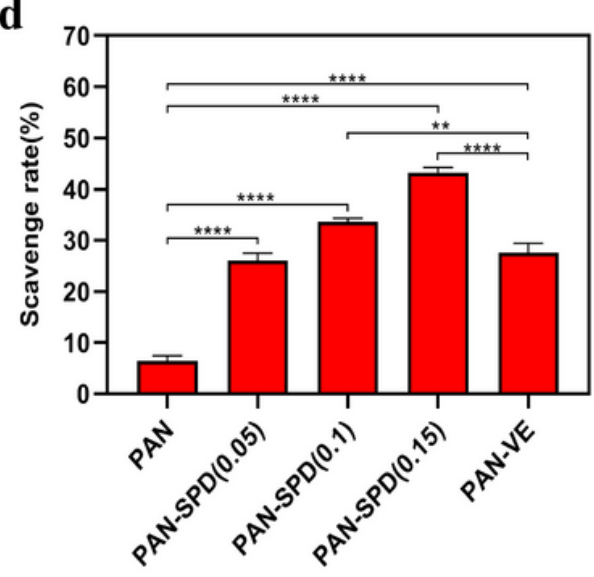

b

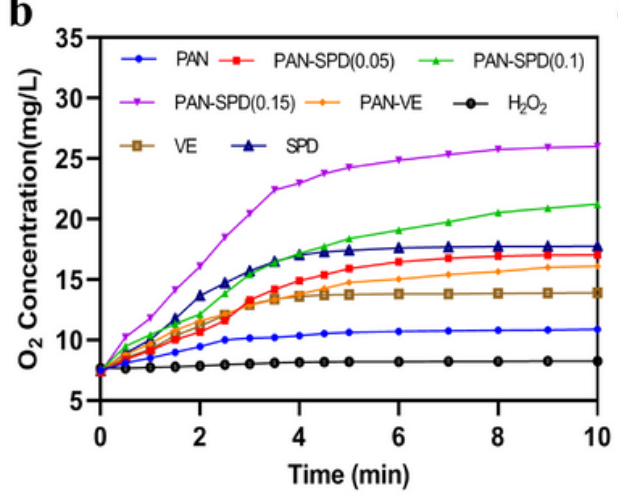

e

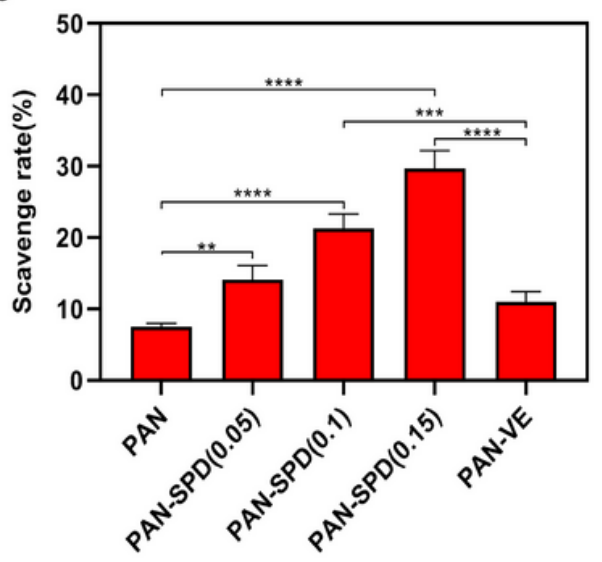

c

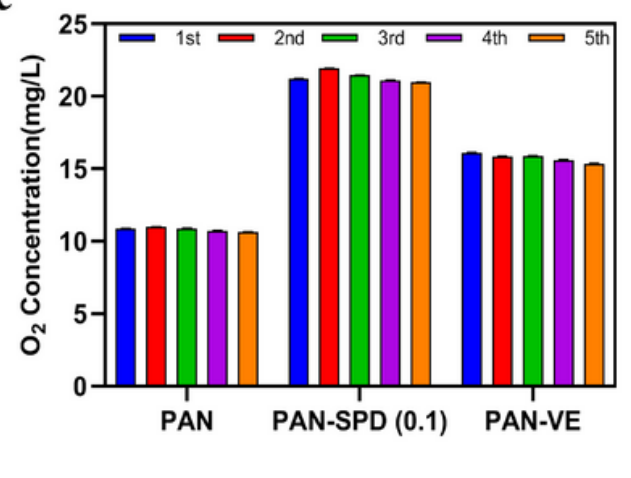

f

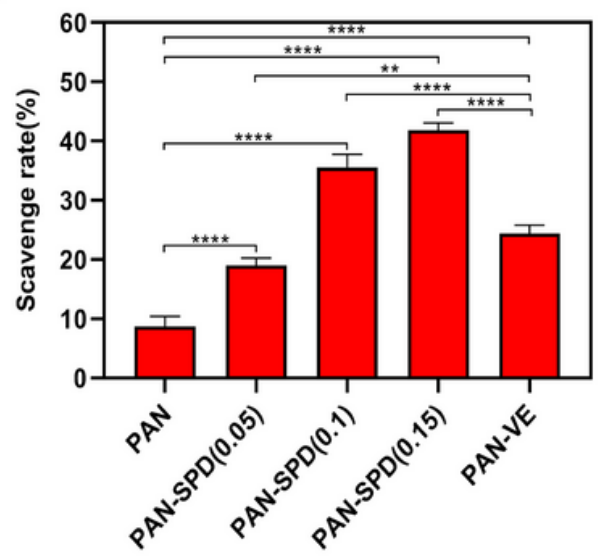

Figure 2

The antioxidant activities of PAN-SPD nanofibers. (a) ABTS • scavenging capacity of PAN, PAN-SPD (0.05), PAN-SPD (0.1), PAN-SPD (0.15) and PAN-VE nanofibers. (b) $\mathrm{H}_{2} \mathrm{O}_{2}$-scavenging capacities of PAN, PAN-SPD (0.05), PAN-SPD (0.1), PAN-SPD (0.15) and PAN-VE nanofibers. (c) $\mathrm{H}_{2} \mathrm{O}_{2}$-scavenging capacities of PAN, PAN-SPD (0.1) and PAN-VE nanofibers in 5 cycles. (d-f) The capability of PAN, PAN-SPD (0.05), PAN-SPD (0.1), PAN-SPD (0.15) and PAN-VE nanofibers to scavenge superoxide anion, hydroxyl radicals and nitric oxide. All data points represent mean \pm S.D. $(n=3)$. *** $P<0.001$, ** $P<0.01$, * $P<0.05$. 

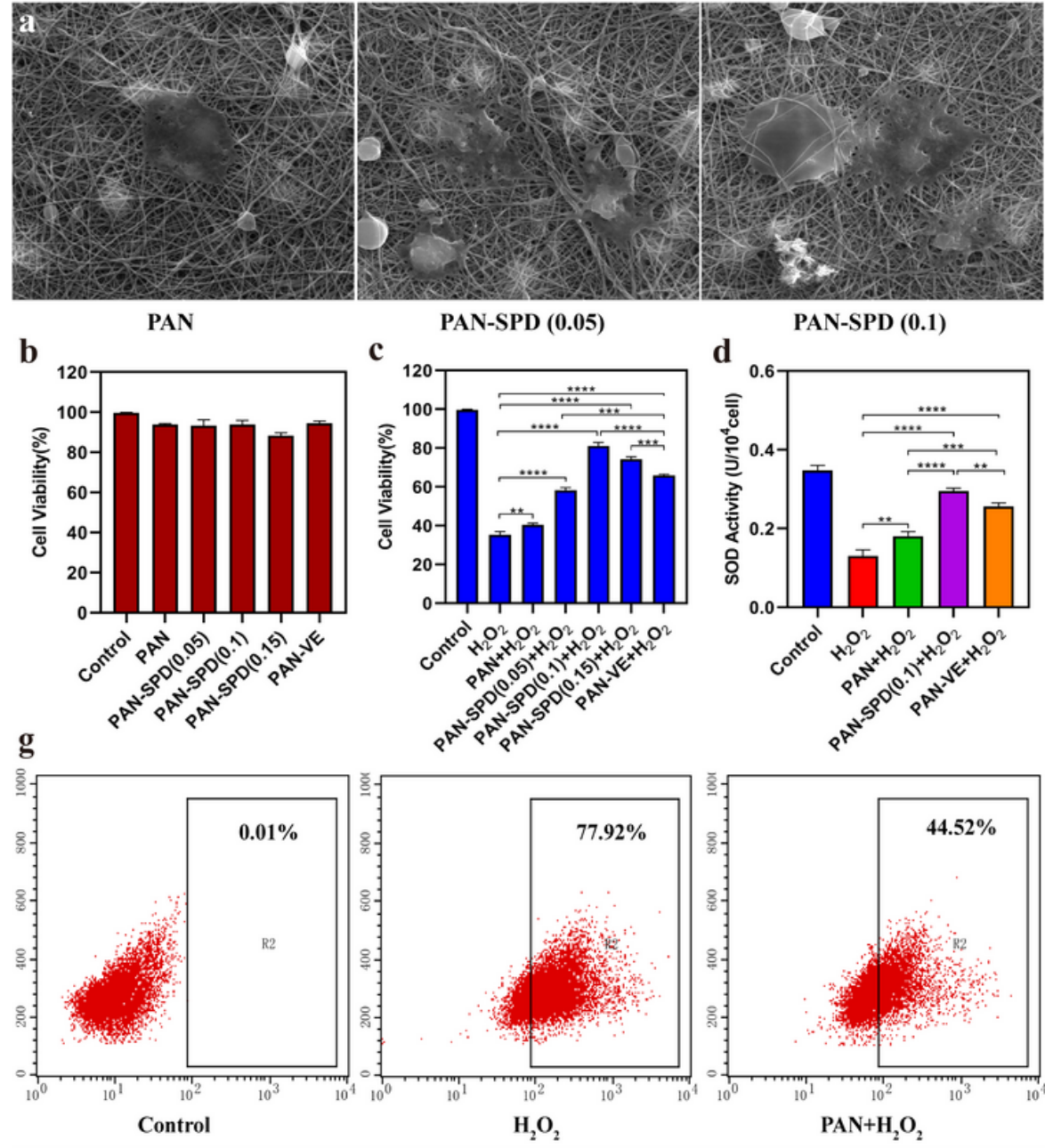

DCFH-DA
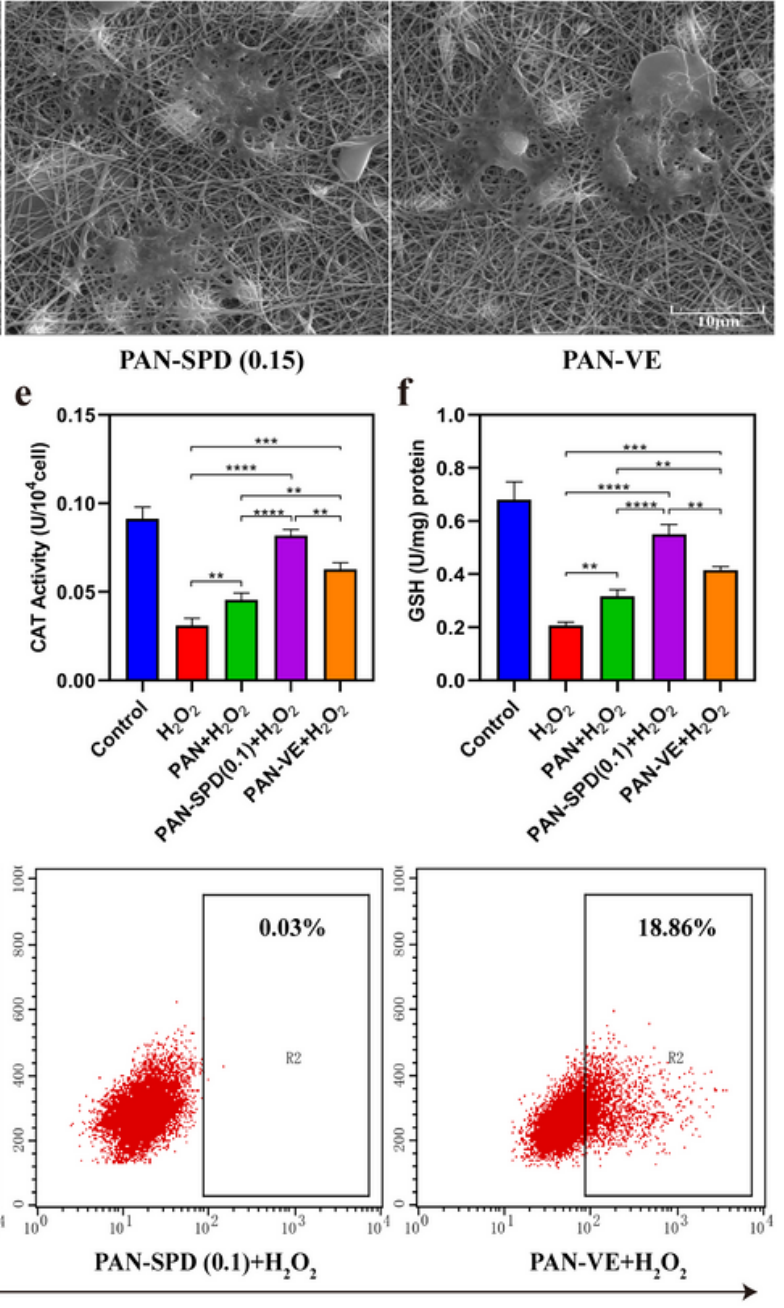

Figure 3

(a) Adhesion of chondrocytes on different groups of nanofibers. (b) Cell viability on PAN, PAN-SPD (0.05), PAN-SPD (0.1), PAN-SPD (0.15) and PAN-VE nanofibers detected by CCK-8 assay. (c) Cell viability on PAN, PAN-SPD (0.05), PAN-SPD (0.1), PAN-SPD (0.15) and PAN-VE nanofibers after treatment with $\mathrm{H}_{2} \mathrm{O}_{2}$ detected by CCK-8 assay. (d-f) Levels of SOD, CAT and GSH. All data points represent mean \pm S.D $(n=3)$.

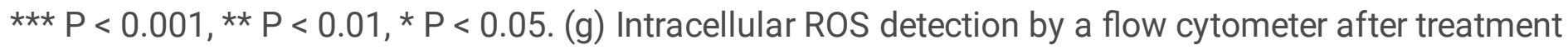
with or without $\mathrm{H}_{2} \mathrm{O}_{2}$ for $24 \mathrm{~h}$. 
a
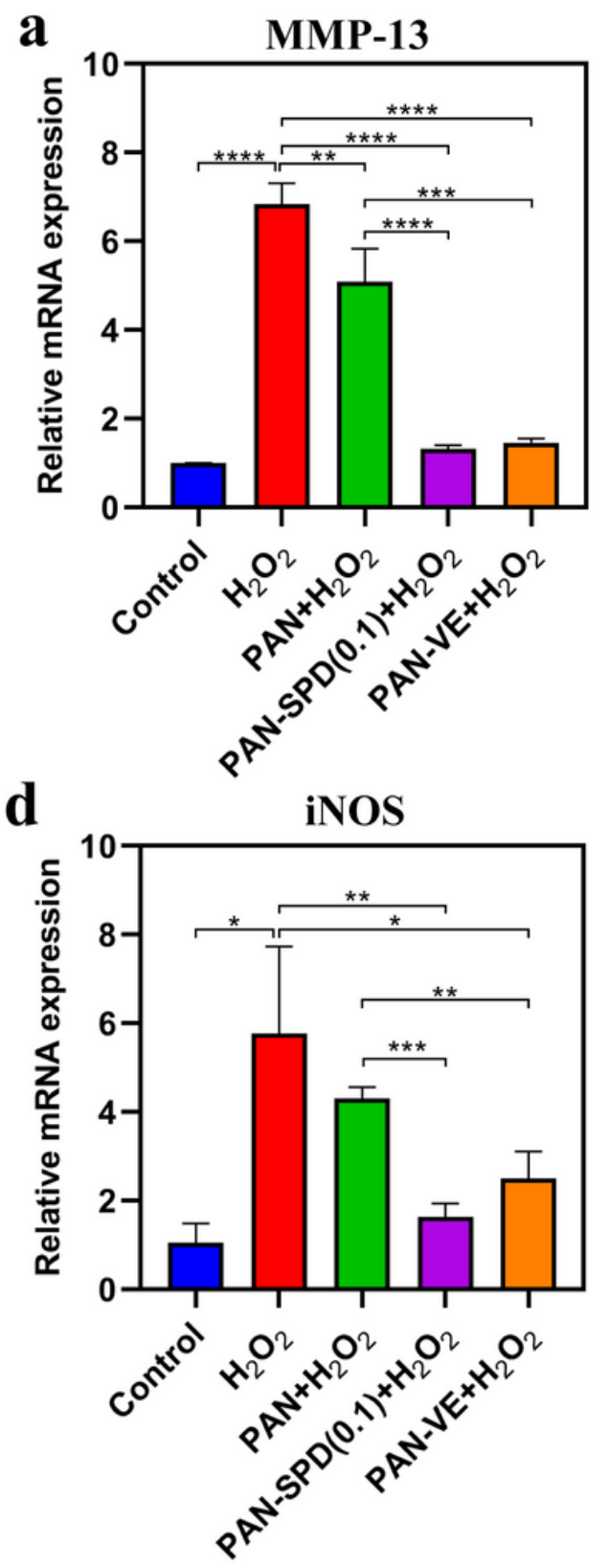

b
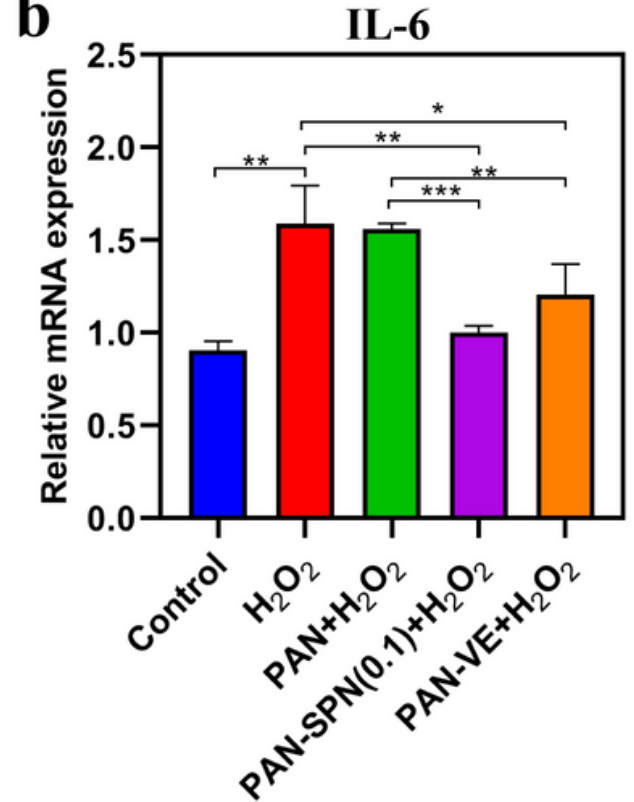

e

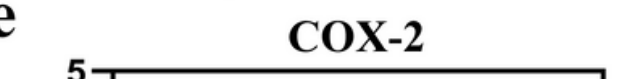

c
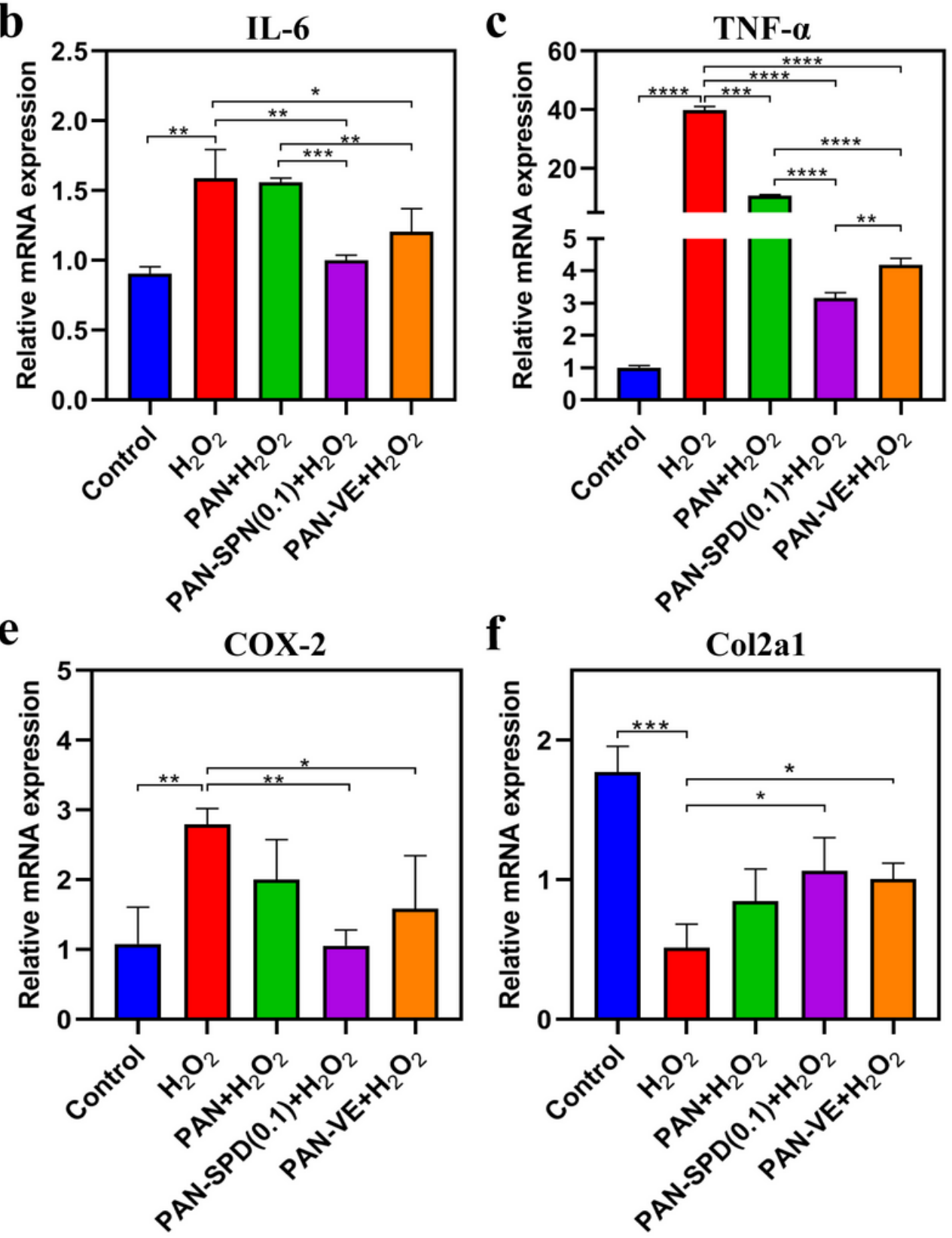
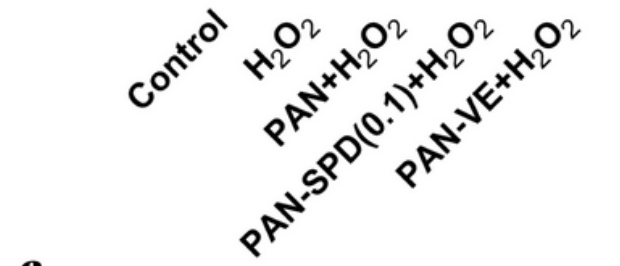

f
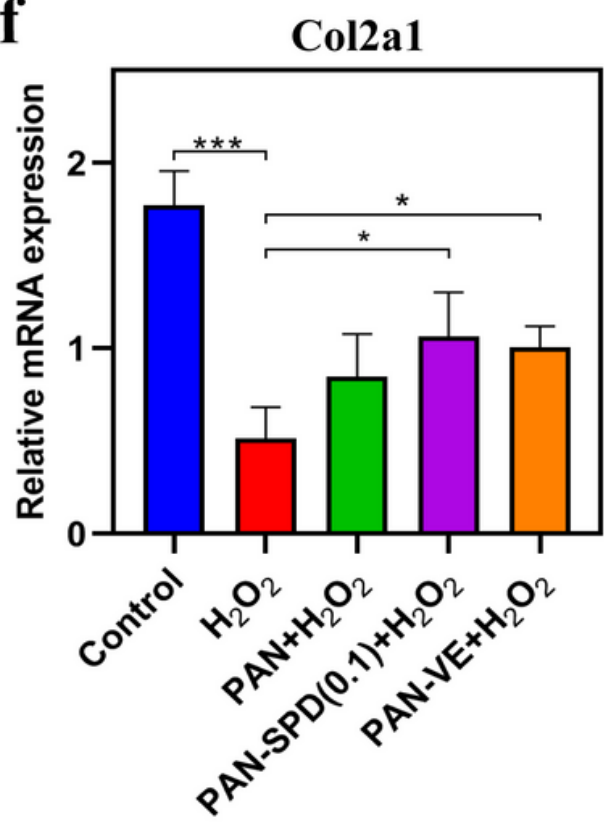

Figure 4

(a-f) qRT-PCR detection the gene expression levels of MMP-13, IL-6, TNF-a, iNOS, COX-2, Col2a1. All data points represent mean $\pm S . D(n=3)$. *** $P<0.001$, ** $P<0.01, * P<0.05$. 


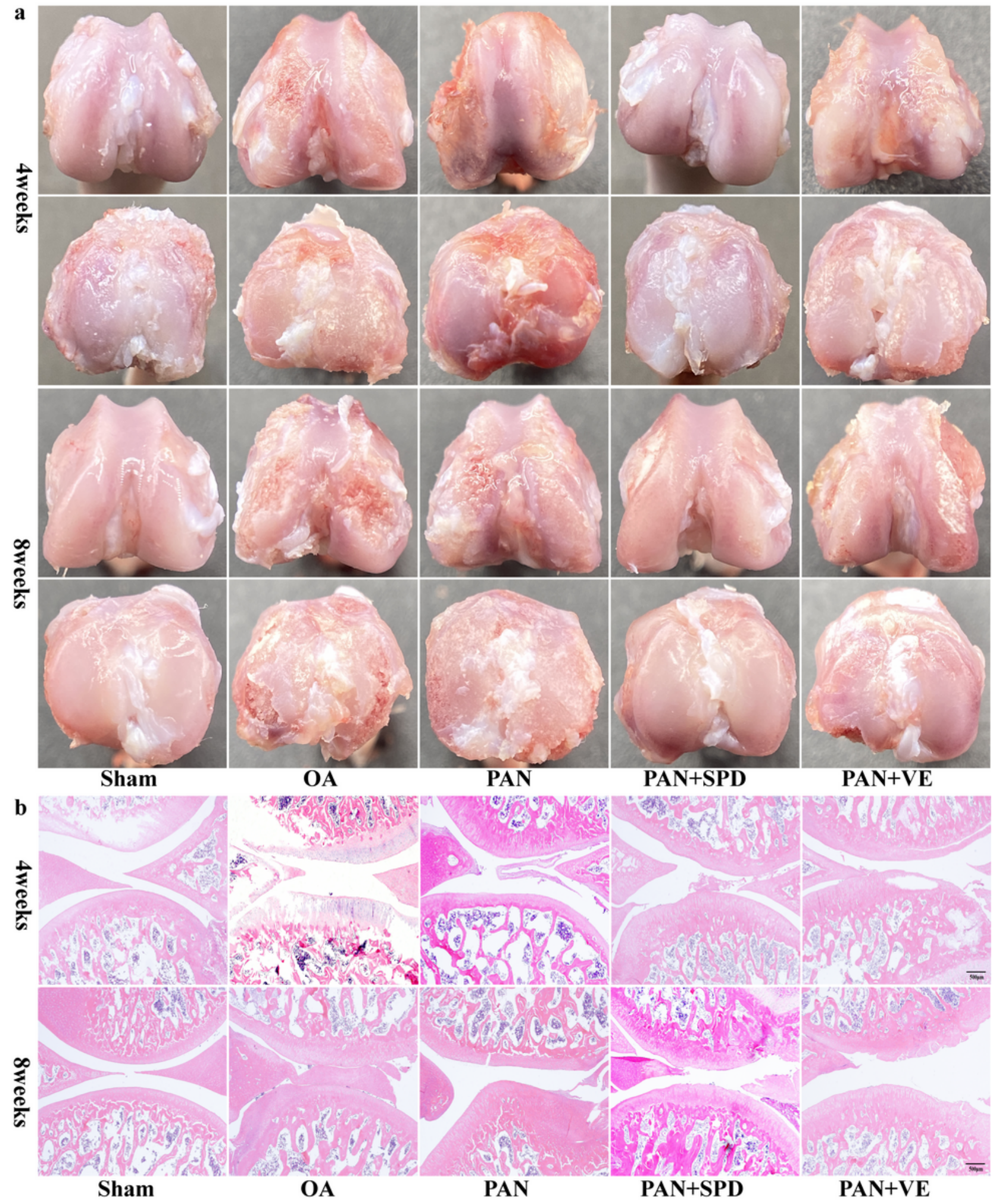

Figure 5

Attenuation of OA progression by PAN-SPD nanofibers in vivo. (a) Macroscopic observation of knee joint after implanted nanofibers for 4 and 8 weeks. (b) HE staining to evaluate degeneration and damage for 4 and 8 weeks. 

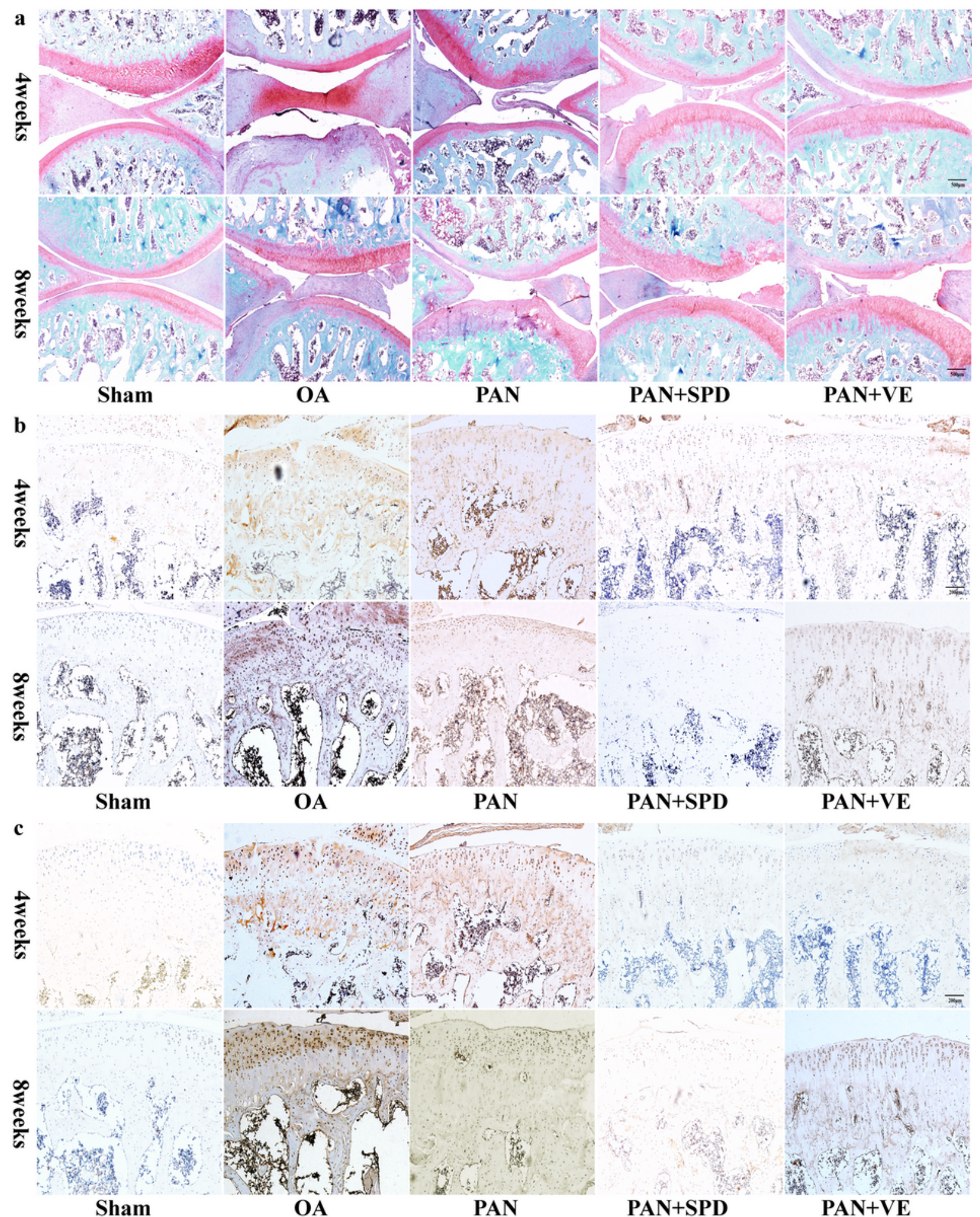

Figure 6

(a) safranin 0 staining to evaluate degeneration and damage for 4 and 8 weeks. (b) and (c) The protein expression levels of inflammatory factors (MMP-13 and IL-6) in the cartilage were detected by immunohistochemical staining assay for 4 and 8 weeks.

\section{Supplementary Files}


This is a list of supplementary files associated with this preprint. Click to download.

- Graphicalabstract.jpg

- Fig.S1.tif

- Fig.S2.tif

- Fig.S3.tif

- TableS1.doc

- Tables2.doc

- Scheme1.png 